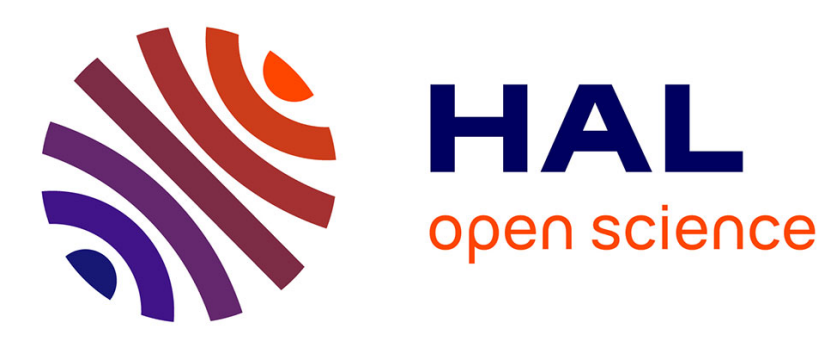

\title{
Approche narratologique des combinaisons audio-visuelles
}

François Jost

\section{To cite this version:}

François Jost. Approche narratologique des combinaisons audio-visuelles. Vibrations. Musiques médias sociétés, 1987, 4 (1), pp.42 - 57. 10.3406/vibra.1987.980 . hal-01796119

\section{HAL Id: hal-01796119 \\ https://hal.science/hal-01796119}

Submitted on 19 May 2018

HAL is a multi-disciplinary open access archive for the deposit and dissemination of scientific research documents, whether they are published or not. The documents may come from teaching and research institutions in France or abroad, or from public or private research centers.
L'archive ouverte pluridisciplinaire HAL, est destinée au dépôt et à la diffusion de documents scientifiques de niveau recherche, publiés ou non, émanant des établissements d'enseignement et de recherche français ou étrangers, des laboratoires publics ou privés. 


\section{Approche narratologique des combinaisons audio-visuelles} François Jost

\section{Citer ce document / Cite this document :}

Jost François. 4. Approche narratologique des combinaisons audio-visuelles. In: Vibrations, N. 4, 1987. Les musiques des films. pp. 42-57;

doi : 10.3406/vibra.1987.980

http://www.persee.fr/doc/vibra_0295-6063_1987_num_4_1_980

Document généré le 25/10/2016 


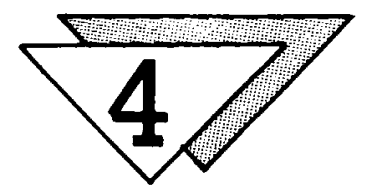

APPROCHE NARRATOLOGIQUE DES COMBINAISONS

AUDIO-VISUELLES, PAR FRANÇOIS JOST

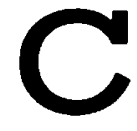

e n'est pas le moindre paradoxe de la sémiologie du cinéma que d'avoir pris pour objet - à l'origine - le film narratif et de s'être occupée en priorité de questions de signification. Pendant longtemps, l'image mouvante n'a en effet été pensée que comme une combinaison de codes, un paradigme de paramètres, et l'on peut presque dire qu'il a fallu les progrès de la linguistique de l'énonciation pour que soient prises en compte par les théoriciens du cinéma les catégories fondamentales du récit comme la narration, le point de vue, etc.

Au moment d'écrire sur le son, ces remarques, que j'ai déjà eu l'occasion de formuler, me viennent en tête. Ne pourrait-on pas reprocher aux analyses sémiologiques de la bande sonore d'avoir négligé, d'une façon similaire, d'envisager les corrélations entre le monde acoustique et les instances du récit ${ }^{1}$ ? Cette question, je me la pose aujourd'hui, relisant notamment la typologie des combinaisons audio-visuelles que j'ai contribué à élaborer. Dans Nouveau cinéma, nouvelle sémiologie ${ }^{2}$, nous proposions en effet, avec D. Chateau, de classer les occurrences sonores de la façon suivante:

- liée-concrète: lorsque le contexte visuel du son inclut la représentation de la source ad hoc. Cette situation se produit chaque fois que nous sommes en présence d'un son in, mais aussi, quand un son, sans être localisable dans l'image, est pourtant présupposé par elle: ainsi, lorsque nous entendons un gazouillis d'oiseau sur un plan de forêt ; 
- liée-musicale: si un son musical est substitué au bruit qui aurait, à sa place, produit une combinaison liée-concrète : un glissando au lieu d'un grincement de porte;

- libre-concrète: un bruit aisément dénoté apparaît dans un contexte où ne lui correspond aucun énoncé iconique adéquat :

- libre-musicale: un son musical est entièrement abstrait par rapport à tout environnement dans le film.

Rajoutons, pour l'intelligibilité de ces définitions, que concret qualifie pour moi : les sons qui dénotent une source sonore non musicale. Cette signification me sépare de Chateau pour qui

par son concret, on entend donc tout objet sonore qui, en l'une au moins de ses occurrences, s'est trouvé lié à un objet ou à un mouvement visualisés, appartenant à l'univers diégétique ${ }^{3}$ du film et susceptibles d'être à l'origine d'un son entendu 4 .

La difficulté première de cette classification, dont la visée était d'abord de se substituer à la vieille opposition in/off, est de déterminer comment le spectateur sait qu'un bruit appartient ou non à la diégèse. C'est en substance la critique formulée par Odin. Après avoir montré que ce procès d'ancrage suppose une connaissance des codes de désignation des sources sonores, il parvient à la conclusion suivante: "Autrement dit, dans un monde donné, il existe un certain nombre de règles précisant ce qui peut être considéré comme la source du son entendu $»^{5}$. Mais, pour être certain que la possibilité théorique de la source sonore est "actuelle», il faut encore qu'elle soit "manifeste », c'est-à-dire qu' « elle manifeste par un mouvement visible dans l'image son statut de source en train d'émettre " 6 . Comme pour Chateau, l'ancrage est attesté in fine par le visuel. Est-ce à dire que la liaison audiovisuelle passe inévitablement par le son in? Ce serait, me semble-t-il, une conclusion fort décevante.

Ecoutons ce passage d'Élisa, vida mía (C. Saura, 1977) pour y voir (?!) plus clair : l'héroïne découvre une amie, allongée sur un lit, morte; dans le lointain on entend des bruits de marteau que rien ne justifie. Pas de justification directe, puisqu'aucune source visuelle n'est redondante ni même en synchronisme avec le rythme des coups; pas de justification indirecte non plus, si l'on accepte la définition que nous propose Chateau : 
la source de production n'est pas clairement visualisée, mais simplement en consonnance avec le contexte visuel (celui qui figure dans le cadre): les chants d'oiseaux et le pic-vert - ces animaux ne sont jamais représentés, leur bruit convenant néanmoins à l'ambiance de la forêt? .

Alors quel est le statut de cette piste sonore? Contrairement au pic-vert qui convient au décor de la forêt, ces coups répétés n'ont aucune relation de "consonnance» avec le décor; on dirait volontiers qu'ils sont en occurrence libre; pourtant ils ne sont pas tout à fait incongrus dans ce décor urbain, et on hésiterait à dire qu'ils sont étrangers à la diégèse car, s'il est impossible de vérifier qu'ils sont émis par une source attestée par l'image, rien ne s'oppose non plus à leur existence dans ce monde.

En revanche, si dans une cellule de prison parisienne, on entend tout à coup une corne de brume, cette intervention sonore s'émancipe de l'univers carcéral et acquiert une liberté d'un autre ordre. L'absence de justification, même indirecte, d'un bruit ne signifie donc pas qu'il soit ipso facto étranger à la diégèse. Si les bruits de marteau sont crédibles, c'est que, somme toute, ils peuvent provenir d'un chantier proche, tout à fait imaginable dans un espace urbain; à l'inverse je ne peux croire que la sirène d'un cargo appartienne à la géographie parisienne. Le statut que l'on confère au son est donc dépendant, non pas seulement des éléments de preuve que peut manifester le film, mais des hypothèses sémantiques qu'il suggère et du savoir "encyclopédique » du spectateur. Encore faut-il nuancer cette dernière assertion: si je ne connais pas Paris - et que j'ignore même qu'il ne s'y trouve pas de port maritime - interpréterai-je cette sirène comme une occurrence libre ? Cette question est une incitation à prendre en compte de nouveaux critères.

\section{Effet/ambiance}

En premier lieu, l'impression ressentie sera différente selon que ce son sera mêlé ou non à d'autres bruits associés à la mer (vagues, mouettes, vent, etc.). S'il intervient de façon isolée, c'est son «irréalisme » qui frappera le spectateur. Les cinéastes 
connaissent bien cette règle que les sémiologues ont tendance à oublier : un bruit (surtout s'il est ponctuel, vertical ${ }^{8}$ s'intègre $^{\prime}$ d'autant mieux à un univers visuel qu'il participe d'une «ambiance »sonore. En ce sens il est vain de vouloir théoriser simultanément l'occurrence unique d'un son concret et une combinaison sonore conçue comme un continuum. La difficulté de l'ancrage de "l'effet » tient fréquemment à son défaut d'iconicité : isolés de leur représentation à l'image, certains sons deviennent incompréhensibles ou ne sont plus perçus. Ainsi, au début de L'homme qui ment, on entend une sorte de vibration curieuse qui pourrait être produite, semble-t-il, par les rebonds d'une balle de ping-pong. Dans le contexte de ces premières séquences où Trintignant arrive au village, ce son est moins senti comme l'occurrence libre d'un son concret que comme une occurrence purement musicale. Ce n'est que bien plus tard en voyant et en entendant la lame de sabre se planter dans un billot que le spectateur comprendra (peut-être) qu'il s'agissait en fait de l'occurrence libre d'un son concret (j'ai fait récemment le même type d'expérience en projetant un de mes films dans lequel on entend à espaces réguliers tourner le barillet d'un revolver qu'on ne voit jamais; le résultat est simple: peu de gens perçoivent ce cliquetis pourtant caractéristique et personne ne l'identifie) ${ }^{9}$. "L'ambiance ", en tant que conglomérat sonore, dénote plus facilement. Bien souvent, ce qui la caractérise, ce n'est pas qu'elle convient au visuel ou qu'elle semble en émaner, c'est plutôt qu'elle n'est pas contradictoire avec l'idée que l'on se fait du monde diégétique posé par la fiction. C'est en fonction de ce vraisemblable sonore que le cinéaste ou le monteur opère ses choix: comme il existait des musiques de poursuite, d'amour, etc. à l'aube du cinéma, il est des atmosphères sonores pour bureau, commissariat, rue, plage, etc. dont la qualité essentielle est la neutralité. Parce que l'univers sonore échappe souvent à la vérification, contrairement à l'image, le son est déterminant dans la construction de la diégèse. Sur des plans de maisons campagnardes répétés à plusieurs reprises dans un film, j'avais placé le bruit continu d'une roue de moulin à eau. Cette association audio-visuelle était évidemment tout à fait libre (aucun cours d'eau n'était à proximité du lieu réel de tournage ou n'était supposé par la fiction) et ne répondait qu'à des nécessités thématiques. Or, force est de constater que jamais aucun spectateur ne met en doute que cette ambiance émane du strict voisinage du décor. 
Probablement aurait-on pu mettre aussi bien des vagues, des grillons, des voitures, etc... quel bruit aurait-il fallu choisir pour provoquer une incompatibilité diégétique?

Si la marge de mancuvre en matière de montage est extrêmement grande, c'est qu'un postulat de sincérité règle la lecture: l'on peut admettre que l'image est manipulée (tout le monde a au moins entendu parler des effets spéciaux), mais on imagine moins volontiers que le son soit l'objet de trucage ou tout simplement de travail. Ainsi, certains spectateurs du Territoire des autres (F. Bel, C. Vienne, M. Fano, 1971), entendant le bruit régulier des roues d'un train sur l'image d'un lièvre courant dans un champ, ont préféré penser que la prise de vue était faite depuis un wagon plutôt que de croire à une association libre, non motivée par une relation iconique ... cela sans penser un instant aux vitesses et aux mouvements relatifs de l'animal et de la machine qu'aurait supposés un tel filmage. Pour gommer l'incongruité sémantique, on est donc prêt à lier n'importe quel bruit à l'espace off. Dans ces conditions, des continuums extrêmement hétérogènes peuvent être pris pour ambiance et liés aux mêmes séquences visuelles. Quels sont les moyens dont on dipose pour rapprocher ou éloigner ces agrégats sonores du présent de la diégèse (car c'est bien en fonction de cette simultanéité que se construit l'autonomie ou l'indépendance de la piste sonore) ? Deux critères sont à prendre en compte: la fréquence textuelle de l'occurrence et sa place dans le récit.

\section{Fréquence textuelle}

Vous êtes cinéaste, vous avez tourné une scène dans la rue: deux personnages sortent d'une gare et prennent un taxi. Vous voulez un fond sonore qui authentifie le décor urbain. Qu'allezvous faire ? Monter le bruit d'une quelconque voiture qui passe et un klaxon dans le lointain? Pourquoi pas ? Le sémiologue parlera alors d'occurrences liées de sons concrets. Imaginez à présent qu'à chaque fois que vos personnages se trouvent dans la rue, on entende exactement la même combinaison sonore. Si l'on considère isolément et abstraitement ces séquences on pourra dire que, dans tous les cas, l'ambiance est liée. Pourtant, pour le spectateur attentif, l'effet de cette liaison se modifiera 
progressivement. Si la première occurrence participe de la construction d'un univers urbain, la seconde diminuera d'un degré la relation mimétique de la diégèse et de notre monde, quant aux suivantes, elles conduiront à mettre en doute tout réalisme sonore et à nier au décor la faculté d'émettre les bruits entendus.

Hypothèse d'école? Non: les Dames du bois de Boulogne (R. Bresson, 1945). D'une façon similaire, lorsque notre ambiance de chantier d'Élisa, vida mía intervient une nouvelle fois, au moment où le père est près de mourir, la liaison audio-visuelle, bien que possible, perd de sa vraisemblance (le lieu de l'action a en effet changé). A l'évidence, on veut nous faire comprendre que la fiction évolue vers la mort. Qui " on " ? Toute la question est là. Ce pronom indéfini surgi au détour d'une phrase nous ramène au cœur du problème de la classification sémiologique des sons. Si, à la première écoute, le klaxon des Dames ... ou les marteaux d'Élisa ... sont liés aux énoncés visuels, à la seconde ils révèlent manifestement un jeu musical qui dévoile plutôt le réalisateur, monteur, musicien, ou, plus génériquement, celui que nous appelons le "grand imagier». Occurrence liée, soit. Mais par qui ou par quoi ? L'identification de l'opérateur de liaison est déterminante pour la construction du récit. Selon qu'elle est unique ou réitérée, la signification narrative de l'occurrence peut en effet changer : dans le premier cas, l'accent sera mis sur la coréférentialité des univers sonore et visuel et l'unicité sera la garantie de l'authenticité (cette correspondance fonde la conception bazinienne de l'image sonore); dans le second, l'illusion mimétique s'érodera et l'accent sera mis sur le récit comme dispositio et sur l'énonciation. Pour que l'ancrage se produise, il n'est pas nécessaire que la source "manifeste par un mouvement visible dans l'image son statut de source en train d'émettre» (Odin). C'est le geste d'un personnage, par exemple, qui peut jouer comme opérateur de liaison. Qu'un personnage tourne la tête au moment où retentissent les coups d'une horloge et l'on comprendra aisément que ce son est en occurrence liée. La compréhension des relations audio-visuelles à l'intérieur du film de fiction ne peut donc faire l'économie d'un raisonnement prenant en compte les instances diégétiques.

Dans cette optique narratologique, il faut préciser les définitions que nous avons avancées, en fonction de leur place dans le récit. 


\section{Occurrences libres, occurrences liées, sens de lecture}

Un homme marche dans la campagne. On entend une "ambiance-café ». A n'en pas douter, la compréhension d'une telle combinaison audio-visuelle sera différente selon (I) que l'on a déjà vu ce personnage dans un café, (II) qu'il vient d'en quitter un, (III) qu'il va y pénétrer, (IV) qu'il ne fréquentera jamais au cours de l'histoire un débit de boisson.

En (II) l'ambiance, d'abord liée, deviendra libre, alors qu'en (III) à l'inverse, elle s'ancrera a posteriori. (I) et (IV) seront à juste titre considérés comme des occurrences libres, mais leur statut narratologique sera différent: la première configuration renverra sans doute à un souvenir, une réminiscence, la seconde à une imagination.

Pour caractériser l'opposition entre (II) et (III), j'ai proposé ailleurs les définitions suivantes:

- occurrence déliée: lorsqu'un son en occurrence liée se continue dans un contexte où ne lui correspond plus le contexte visuel qui avait suscité l'ancrage (on se délie alors du réalisme comme on se délie d'une promesse);

- occurrence captée : lorsqu'un son en occurrence libre s'ancre après coup à une source sonore. Ce peut être le cas (III), mais ce peut être aussi l'ancrage d'un son par un indice visuel, comme, par exemple, lorsque le geste d'éteindre une radio entraîne l'arrêt de la musique de film. Exemplaire de cette soumission du sonore à l'indiciel est cette scène de Glissements progressifs du plaisir ( $\AA$. Robbe-Grillet, 1974): le magistrat parvient à la cellule de l'héroïne : à travers la porte il entend des cris, des plaintes comme si on torturait la jeune femme. Occurrence liée, se dit pour lui-même le sémiologue. En ouvrant la porte, l'homme de loi va découvrir une de ces scènes que Robbe-Grillet affectionne, poursuit le théoricien. Surprise : en pénétrant dans la cellule on découvre la jeune femme, nue, dans une attitude dont le calme est à l'opposé de la bande sonore. Occurrence libre? Le magistrat se dirige alors vers le tourne-disque, soulève le bras de l'appareil et les cris cessent aussitôt : il s'agit bien d'une occurrence captée, puisqu'un geste la lie à une source sonore, inédite il est vrai et quasi imprévisible. 


\section{Indices visuels et ancrage sonore}

Revenons sur (I) et (IV). A nouveau, on s'aperçoit que la signification de l'occurrence dépend de l'opérateur de liaison. Lorsque, dans Hiroshima, mon amour, on continue à entendre la musique du bar japonais sur les souvenirs de Nevers, plusieurs critères nous permettent de comprendre que ce choix musical traduit le point de vue auriculaire de l'héroïne (je parle alors d'auricularisation interne secondaire). a) le fait que nous l'avons vue dans ce lieu avant l'intervention des images du passé ; b) la disparition progressive de cette ambiance à mesure que s'intensifie le travail de la mémoire; $c$ ) son retour lorsque le japonais met fin à l'hallucination par une gifle.

Rappelons que le narrateur d'un récit peut choisir de se situer partout et nulle part, adoptant un regard divin, de voir les événements de son point de vue ou par les yeux d'un personnage. S'agissant d'un medium qui mobilise deux perceptions la vue et l'ouie -, la possibilité d'adopter tel ou tel point de vue est double: il faut choisir une place de la caméra et une "perspective » sonore. Pour désigner ces deux problèmes, j'ai proposé de parler d'ocularisation (= point de vue visuel) et d'auricularisation (= "point de vue " sonore). Je parle "d'auricularisation interne secondaire » lorsque la subjectivité auditive est construite par le montage et le visuel (geste du personnage, changement d'intensité en relation avec le déplacement d'une source sonore attestée à l'image), "auricularisation interne primaire ", lorsque certaines déformations écartent la bande sonore des codes du réalisme et renvoient d'emblée à la subjectivité d'une oreille. "Auricularisation zéro", lorsque le son n'est relayé par aucune instance diégétique (narrateur ou personnage). L'ensemble de cette théorisation est explicité dans l'Eil-caméra. Entre fil et roman (titre provisoire), à paraître aux PUL. Le contexte visuel et l'ordre des décors jouent donc un rôle majeur dans la lecture du film : si le son gagne progressivement son autonomie, je n'oublie jamais en effet la source à laquelle il est ancré. De la sorte, ce qui est entendu se mue en écouté : si la musique disparaît soudain, c'est que les personnages n'y font plus attention.

Cette disparition progressive est bien différente du "shuntage » qui facilite parfois le passage d'une ambiance à une autre: lorsqu'un personnage sort d'un café et que l'on entend encore, 
disparaissant, quelques verres qui s'entrechoquent et la musique d'un juke-box, cette atténuation qui déborde légèrement sur un décor hétérogène renvoie plutôt au geste du "grand imagier » qui a pu vouloir ce chevauchement pour de multiples raisons (de la même façon, il devient de plus en plus fréquent de monter des sons en anticipant légèrement sur la représentation de leur source pour créer de micro-surprises par une captation retardée).

Seul peut être dit "libre " le son qui n'est lié au contexte ni en amont ni en aval. Par définition l'occurrence libre d'un son ne peut appartenir à l'univers perceptif du personnage (sinon il serait lié au décor). Toutefois, deux cas sont à distinguer : soit elle participe de son univers mental, soit elle lui échappe totalement, n'étant qu'une manipulation de l'énonciateur. Or, il faut bien reconnaître que la confusion entre ces deux ancrages est moins répandue qu'on peut le croire a priori. Car, si j'excepte le film de photos qui pose des problèmes spécifiques, le traitement de la bande sonore, comme le comportement des personnages, permet de savoir si le son doit être ou non lié à une instance diégétique. Si en théorie on peut affirmer que rien ne distingue une voix off d'une voix intérieure, dans une salle de cinéma aucun spectateur ne confondra l'une avec l'autre. Le jeu de l'acteur, sa plus ou moins grande présence, le mixage différencieront sans difficulté ces deux voix. De la même façon, on néglige à tort dans la typologie des combinaisons audio-visuelles le rôle de tous ces paramètres sonores dans l'ancrage du son à la diégèse. Peu de films vont à l'encontre des conventions qui règlent la représentation d'un souvenir ou d'une imagination auditive: éloignement, discontinuité, entrée et disparition progressives des bruits, etc. Même un film comme L'homme qui ment, qui constitue sans doute l'une des recherches les plus élaborées de $\mathrm{M}$. Fano, respecte ces codifications lorsqu'il s'agit d'exprimer un rêve de Boris. A ce traitement particulier du sonore, le cinéma narratif combine l'attitude du personnage : ce n'est pas seulement la contradiction d'une série de bruits avec le profilmique ${ }^{10}$ qui la fait glisser vers l'occurrence libre, c'est aussi que le personnage est de trois quart, les yeux perdus dans le vague, etc. En effet, le propre de l'occurrence liée d'un son concret écouté par un personnage, c'est d'être latéralisé et localisé au sens où "la localisation d'un son est la perception d'un son et la détermination qu'il a origine dans un point de l'espace choisi parmi l'infinité des directions possibles (en haut, 
en bas, en avant ou en arrière) ${ }^{11}$; le bruit imaginé ou souvenu, à l'inverse, échappe à toute localisation : il vient d'ailleurs, du lointain dans lequel se perd le regard du personnage (la fixité entre alors en paradigme avec la mobilité corporelle qu'on associe généralement à "l'écoute active » qui caractérise l'opération de repérage d'un son).

Ces indices sonores et visuels, qui ancrent le bruit à une instance diégétique, jouent comme des opérateurs de modalisation et font glisser l'occurrence vers l'auricularisation interne. Si l'occurrence libre d'un son concret n'est ni modalisée par la manipulation acoustique ni relayée par un geste du personnage, on aura tendance à la mettre au compte de l'énonciateur et à la lire comme un motif à fonction plutôt musicale : dans Monsieur Arkadin (O. Welles, 1956), lorsque le pouvoir du personnage joué par Welles vacille, on entend la vibration d'un avion qui part à la dérive, aucune instance profilmique ne remarque ce bruit. Et pour cause : rien, si ce n'est une anticipation thématique, ne motive une telle occurrence; elle n'a sa source ni dans la tête d'un personnage ni dans l'espace limitrophe, elle n'est qu'une indication prémonitoire de l'énonciateur à l'usage du spectateur averti (= celui qui a vu plusieurs fois le film). De la même façon que le personnage n'est pas censé savoir comment il est filmé, il ignore les occurrences libres non modalisées, ce qui leur confère un statut extra-diégétique qui les rapproche du son musical, au moins d'un point de vue narratologique.

\section{Ancrage, synesthésie}

Avec le recul, notre définition de l'occurrence liée-musicale (= son musical substitué à un bruit qui aurait produit à sa place une combinaison liée-concrète) paraît un peu bancale : en effet, alors que l'occurrence liée-concrète est définie, selon un critère sémantique, par la redondance éventuelle du sonore et du visuel, quand on parle de la substitution d'un glissando de violon à un grincement de porte, on ajoute au concept un critère de synchronisme. En toute rigueur, lié-musical devrait donc plutôt définir une musique dont il n'est pas contradictoire de penser qu'elle émane du contexte visuel (orchestre, tournedisque ou radio pouvant la motiver). Ce réajustement termino- 
logique ne doit pourtant pas nous faire oublier que, effectivement, certaines musiques remplacent des bruits que l'on aurait pu entendre: mais, à y bien réfléchir, ce qui caractérise la substitution du glissando au grincement de porte, c'est non seulement la proximité plastique des deux sons, mais surtout des paramètres temporels (en l'occurrence: horizontalité, longueur, rythme). En cela, ce traitement sonore n'est pas éloigné de tout ce que l'on peut regrouper sous le terme générique de ponctuations: qu'il s'agisse d'arrêter la musique sur un geste ou, au contraire, d'accompagner un mouvement de caméra par une note tenue ou, même, de couper une mélodie à la "collure», tous ces traitements sonores, qui jouent sur la temporalité de l'action, le rythme des énoncés visuels ou du montage, sont des occurrences ponctuatives d'un son musical. Ce qui les définit en termes narratologiques, c'est leur extériorité au monde de la diégèse et, partant, le fait qu'elles contribuent à construire une image de l'énonciateur ou du genre cinématographique: ainsi, on opposera la musique burlesque qui ponctue par son rythme propre la cascade des événements à ces scènes où une note verticale provoque un mouvement de tête de l'acteur (dans un cas la musique favorise la reconnaissance du genre, dans l'autre le lien apparent, de cause à effet, entre une occurrence musicale étrangère au contexte visuel et le geste, ouvre une brèche par laquelle le monde diégétique se trouve incroyablement mis en relation avec l'énonciateur - comme dans certains regards à la caméra).

C'est un phénomène du même genre qui se produit dans Le Grand Frisson (M. Brooks, 1978), lorsque le nouveau psychiatre, qui se rend pour la première fois à l'asile qui sera son lieu de travail, donne tous les signes d'une frayeur grandissante en entendant une musique de film digne des films d'Hitchcock. Le paradoxe réside encore dans cette relation entre l'occurrence libre-musicale, tout à fait hors diégèse, du côté de l'énonciateur, et le personnage comme instance diégétique, dans l'énoncé ... comme si les personnages de roman se mettaient à trembler devant certaines descriptions. Or, comme on sait, le gag à double détente dans ce film vient du fait que finalement la voiture du nouveau médecin croise un orchestre qui répète dans un autocar, ce qui a pour effet de capter cette occurrence musicale que l'on croyait libre et qui, in fine, était liée (selon notre nouvelle définition). 
Mais revenons à cette relation plastique qui unit l'occurrence musicale au visuel, que Fano appelle "congruence», et qui s'apparente plutôt à la synesthésie. Si, montant une musique sur une suite de plans, on dit souvent qu'elle "colle» bien, qu'elle "marche » ou "fonctionne », ce n'est pas par simple inaptitude à expliquer le phénomène, c'est plutôt en raison de la conviction que certaines images vont bien avec certains sons, indépendamment des questions de rythme ou de sens. De même que l'arbitraire de la langue n'a jamais empêché les rêveries sur les dérivations mimétiques (je renvoie au remarquable Palimpsestes: Seuil, Paris, de G. Genette), l'arbitraire des signes musicaux se prête particulièrement bien aux correspondances visuelles. On peut regretter que subsiste cette incompréhension quant à la nature des signes, mais on ne saurait nier le rôle déterminant des associations synesthésiques dans la réception filmique (comme on le sait certains ordinateurs fabriquent aujourd'hui des complexes audio-visuels fondés sur ce postulat). Cette acceptation de la synesthésie comme règle implicite de la perception explique en partie le succès des "clips vidéo ». Il est remarquable en effet que ces petits films musicaux mettent en jeu des procédés de montage, des mouvements de caméra, des trucages, des structures narratives même, qu'une certaine modernité cinématographique a utilisé naguère sans succès : par exemple le fait de monter "cut » trois fois le même geste agace souvent le public lorsqu'il s'agit d'un film de fiction des années soixante et est au contraire apprécié dans un "clip » d'Isabelle Adjani. C'est qu'en fait la chanson elle-même, structurée sur la répétition, la reprise ou la variation, autorise et motive une telle équivalence visuelle. En fin de compte, l'occurrence libre musicale, qui nous semblait dévoiler l'énonciateur, finit par naturaliser le récit en effaçant l'idée même d'énonciateur (l'image doit se soumettre au diktat de la musique). L'étude des liens entre synesthésie, ancrage sonore et lecture de l'image reste à faire. Nul doute qu'elle contribuerait largement à renouveler la compréhension de la réception du lacis audio-visuel.

Il n'est plus temps en ce point de notre travail d'ouvrir une piste qui nécessiterait toute une expérimentation. Je me contenterai donc de tirer quelques conclusions de ce retour sur mes propres traces, en m'appuyant sur le tableau de la page 55, qui résume le présent article. 
On constate d'abord qu'une même combinaison audio-visuelle (par exemple une occurrence liée) peut aussi bien renvoyer à l'énoncé, à l'énonciation qu'à une instance du monde diégétique (un personnage). L'attribution d'une occurrence à l'un de ces trois pôles est une opération à la fois beaucoup plus complexe et beaucoup plus simple qu'on le suggère généralement.

Beaucoup plus complexe, parce que l'ancrage d'un son à une image ne mobilise pas seulement des codes de reconnaissance culturels et une attention portée aux sources sonores, mais aussi l'appréhension de multiples paramètres comme le mixage, la manipulation des sons (filtrage, réverbération, etc.) et le mode d'enregistrement (présence, éloignement du micro, "couleur " de l'ambiance sonore, etc.). Par ailleurs, des présupposés règlent l'écoute du spectateur: j'ai évoqué un postulat de sincérité, mais j'aurais pu dire aussi que ce qui sémantise un son, c'est le hasard et la nécessité: plus un bruit paraît aléatoire, plus il joue comme effet de réel, plus en fin de compte il tend vers "l'image sonore» ( $A$. Bazin). C'est le repérage de la régularité qui fait basculer la bande sonore vers l'énonciateur. Le montage en boucle est intéressant à cet égard: si je prends quelques sons - verres qui s'entrechoquent, pas, bruits de meubles, paroles échangées - et que je les fait repasser à plusieurs reprises pendant une scène de cocktail, il sera possible de les prendre pour une ambiance ad hoc tant que l'on n'aura pas identifié un indice de répétition qui les fera basculer vers l'énonciateur.

$\mathrm{Si}$, sur toute la scène, on voit un homme perdu dans ses pensées et qu'une légère réverbération s'ajoute, cette boucle pourra aussi traduire la monotonie qu'il ressent. Une même combinaison audio-visuelle (ici : occurrence liée-concrète) change donc de signification en fonction de la valeur narratologique que l'on peut déduire des indices sonores et visuels. Le sémiologue a parfois tendance à considérer la dialectique audio-visuelle comme des configurations d'occurrences ; le spectateur, de son côté, intègre très vite les conventions qui aident à différencier un bruit imaginé d'un bruit censément réel. Dans ce repérage, le rôle de l'image n'est pas négligeable. Cela n'étonne guère lorsqu'on garde en tête que la localisation d'un son dans l'écoute directe ne peut se faire que dynamiquement : notre identification aux mouvements de l'acteur et aux indices visuels est 
d'autant plus grande que, nous-mêmes, pour choisir entre les directions possibles d'un son, nous combinons des mouvements de têtes et les excitations visuelles (d'où la difficulté à ancrer certains bruits la nuit).

\section{Classification narratologique des combinaisons audio-visuelles}

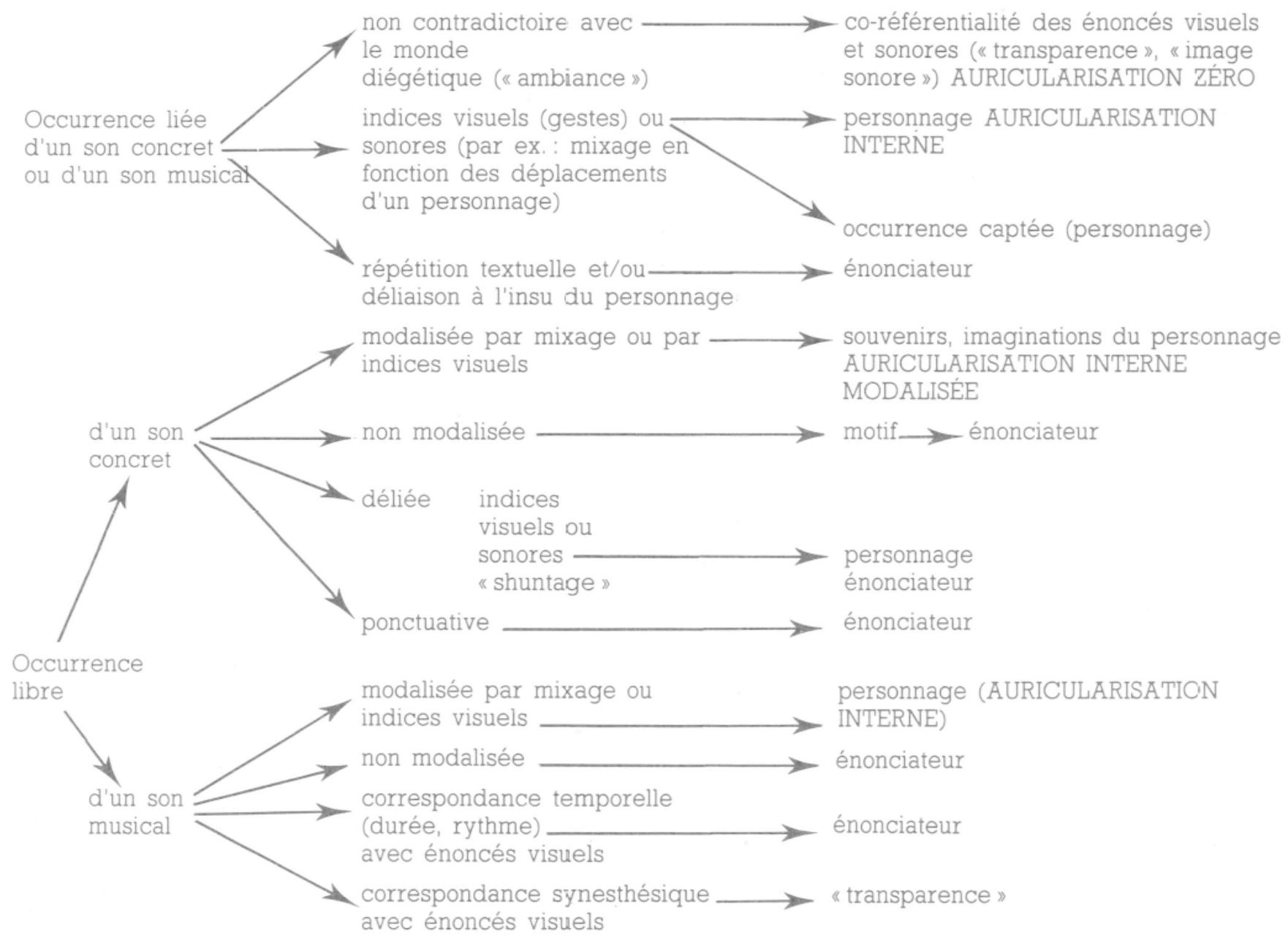

Pour ancrer un son, il n'est donc pas nécessaire que la source soit manifeste ; la lecture des gestes, l'écoute des intensités, des niveaux relatifs, etc. sont dans bien des cas des preuves suffisantes, bien qu'indirectes, de sa production dans un espace contigu.

On perd de vue la simplicité relative de la lecture de la bande sonore quand on oublie qu'elle n'est pas seulement objet de 
montage, mais matière. Prenant pour objet de réflexion les combinaisons audio-visuelles ou les codes nécessaires à l'intellection de ces combinaisons, on raisonne souvent sur un modèle théorique moyen qui laisse en dehors des paramètres comme le timbre, l'intensité, la présence, les niveaux, etc. Or ce sont les variations de ces paramètres qui confèrent une valeur narrative aux sons, car elles ont des effets sur leur ancrage au monde diégétique (sont-ils à l'extérieur ou à l'intérieur?), sur la connaissance du monde mental du personnage (notamment "l'auricularisation "), le degré de réalité qu'il faut accorder aux informations diégétiques (en fonction de leur modalisation), sur leur temporalité.

Toute analyse suppose résolues en amont des difficultés de transcription. On a beaucoup réfléchi sur la description de l'image à travers le découpage et, plus récemment, sur les problèmes posés par le dialogue. Pour étudier la bande sonore, il n'est d'autre alternative que de la considérer comme une partition ou, tout au moins comme une feuille de mixage.

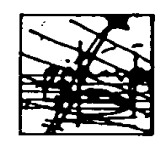

\section{Notes}

1. La sémiologie cinématographique a longtemps pris comme objet les problèmes de signification in abstracto sans s'interroger sur le fonctionnement des paramètres étudiés à l'intérieur d'un récit. Ainsi, pour le son, on a plutôt cherché à classifier les combinaisons audiovisuelles a priori qu'à expliquer les combinaisons en fonction de leur ancrage aux " instances du récit " que sont le narrateur et les personnages.

2. Chateau (D.), Jost (F), Nouveau cinéma, nouvelle sémiologie, U.G.E., 10/18, Paris, 1979, repris par Ed. de Minuit.

3. Diégèse. Rappelons que ce terme a été injecté dans la théorie du cinéma par
E. Souriau (I'Univers filmique, Flammarion, 1953). La sémiologie du cinéma et les théories littéraires l'ont repris ensuite. Selon Souriau: "Diégèse, diégétique: tout ce qui appartient "dans l'intelligibilité" au monde supposé ou présupposé par la fiction du film ". L'univers diégétique comprend donc les événements, les "ingrédients " de la fiction (violence, honneur, amour, etc.) et les "postulats" qui la rendent vraisemblable.

4. Le cinéma comme langage, Université de Paris I, 1976, thèse de $3^{\mathbf{e}}$ cycle, p. 175.

5. Odin (R.), "A propos d'un couple de concepts son in vs son off ", in Sémiolo- 
giques, Presses universitaires de Lyon, p. 122

6. Ibid., p. 124

7. LoC. cit.

8. M. Fano oppose la verticalité à l'horizontalité comme le ponctuel au continu. Un coup bref est vertical ; une onde bourdonnante est horizontale.

9. Au moment de relire cet article, je vois Antonioni à la télévision française, qui commente l'avant-dernier plan de Profession reporter. II fait remarquer qu'on en- tend off, sur le moteur d'une Vespa, une détonation de revolver ... et il déplore que personne ne l'identifie!

10. Profilmique: tout ce qui existe réellement dans le monde, mais qui est spécialement destiné à l'usage filmique; notamment tout ce qui s'est trouvé devant la caméra et a impressionné la pellicule (Souriau, op. cit.).

11. Gemelli, "La localisation des sons" in Miscellanea psychologica Albert Michotte, Vrin, Paris, 1947.

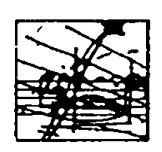

